\section{Practical Blood Transfusion}

By Douglas W. Huestis, Joseph R. Bone and Shirley Busch. Pp. 383, illustrated. Boston: Little \& Brown; London: J. \& A. Churchill, 1970. £7.00.

This book provides a comprehensive account of the problems encountered in setting up and running a Blood Transfusion Service. It includes chapters on immunology, blood transfusion practice and the hazards involved and blood component therapy.

The Donor Service is dealt with in great detail and proper emphasis is placed on the recruitment and choice of donors. The chapter on blood components is well set out and gives useful if brief notes on the uses of the various blood fractions. The present day treatment of haemolytic disease of the newborn is detailed but prophylaxis is barely mentioned. The book ends with a summary of medico-legal problems which fortunately has little relevance in this country.

This book would make good reading for medical students, hospital junior staff and even consultants. It is insufficiently detailed for day to day use by technical staff but provides a good general description of the running of a transfusion service which might be read with profit by supervisory staff in Blood Bank Centres at hospital and regional levels.

\section{Atomic Medicine}

Edited by Charles F. Behrens, E. Richard King and

JAmes W. J. CARPender, 5th edition. Pp. 874, illustrated.

Baltimore: The Williams and Wilkins Company; Edin-

burgh and London: E. \& S. Livingstone, 1970. £13.50.

In its fifth edition this book will consolidate its reputation as the most comprehensive and probably the best book in English on nuclear medicine. The subject is so vast-ranging from nuclear physics, strategy and ethics to almost every branch of clinical medicine and biological research-that one can but marvel at the editorial feat of ensuring both a balanced presentation and a reasonably uniform style. The chapters on basic physics and nuclear strategy by the senior editor remain masterly-though the latter is now almost certainly obsolete. The clinical sections reflect the diverging paths of diagnostic and therapeutic nuclear medicine. Therapeutic nuclear medicine has been virtually stagnant for the past 10-15 years, chemotherapy and other forms of treatment replacing irradiation as the treatment of choice in many fields formerly the preserve of radiotherapy. By con- $z$ trast, diagnostic nuclear medicine has made tremendous $\mathbb{D}$ strides since the last edition (1964); and it remains a field of

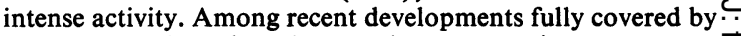
the present volume have been various new stationary-camera- $\overrightarrow{\bar{F}}$ type scanners allowing rapid image production and the use of short half-life radioisotopes. The combination of rapido imaging (making it possible to use larger radioactivities of $\overline{0}$ short-half-life isotopes without increasing patient exposure), $\overline{\bar{\sigma}}$ coupled with the introduction of new compounds and colloids $\vec{\Phi}$ for specific scanning purposes are bringing about a diagnostic $\varrho$ revolution comparable to the introduction of X-ray diagnosis. Many of the contributors to the current edition have been in the forefront of these developments; and the informa- $\vec{O}$ tion they convey is impressively up-to-date. Altogether this is an impressive book as well as an indispensable one.

\section{A Primer of Immunology}

By F. A. WARD. Pp. 130. London: Butterworths, 1970. $£ 2.10$.

Any book that hopes to satisfy the 'practical requirementsơ of Medical Students and Medical Practitioners' and 'prove $\vec{\sigma}$ helpful to Medical Technologists, Nurses and others' runsi the risk of pleasing none of them. Dr Ward's book, more $\vec{f}$ accurately a primer of clinical immunology, is, in fact, a $\frac{1}{\sigma}$ lucid and entertaining introduction to a complex subject and could be read with profit by all these groups. However, the author's aims and the size of the book inevitably mean that the coverage is somewhat eclectic and superficial. On occasions, this leads to the use of unexplained concegts which will tend to confound the beginner. The best chapters are those dealing with various aspects of immunohaema 6 logy which is one of the author's major fields of inter $\triangle$ Other chapters are concerned with antigens, antibodiss. antigen-antibody reactions, immunoglobulins, immediatelo and delayed hypersensitivity, diseases of the immunologica apparatus, autoallergic disease, and immunology in the diagnosis and prevention of disease. For those who wish too study these subjects in greater depth, there are other up-to-음 date introductory books in clinical immunology now available and at substantially fewer pence per page. 